\title{
The Relationship between Chemokine Ligand 3 and Allergic Rhinitis
}

Ovidiu Berghi $^{1}$, Mihai Dumitru ${ }^{2}$, Ramona Caragheorgheopol ${ }^{3,4}$, Catalin Tucureanu ${ }^{3}$, Anca SimioniucPetrescu $^{5}$, Roxana Sfrent-Cornateanu ${ }^{6}$, Calin Giurcaneanu ${ }^{7}$

1. Dermatology, Carol Davila University of Medicine and Pharmacy, Bucharest, ROU 2. Anatomy, Carol Davila University of Medicine and Pharmacy, Bucharest, ROU 3. Immunology, Immunology Laboratory, "Cantacuzino" National Institute for Military Medical Research and Development, Bucharest, ROU 4. Immunology, University of Bucharest, Bucharest, ROU 5. Ambulatory, Sfânta Maria Clinics and Laboratories, Bucharest, ROU 6. Immunology and Pathophysiology, Carol Davila University of Medicine and Pharmacy, Bucharest, ROU 7. Oncologic Dermatology, Carol Davila University of Medicine and Pharmacy, Bucharest, ROU

Corresponding author: Ovidiu Berghi, oberghi@yahoo.com

\section{Abstract}

\section{Background}

Allergic rhinitis (AR) is a chronic and frequent condition characterized by an excessive response of the immune system to innocent substances encountered in the nasal mucosa. These reactions are mediated by many factors, including chemokines. Chemokine ligand 3 (CCL3, a macrophage inflammatory protein $1 \alpha$ ) is a chemokine implicated in the activation of mast cells - white cells shown to be highly involved in orchestrating allergic reactions. The present study evaluated the role of CCL3 in AR.

\section{Material and methods}

Thirty-nine participants, including 24 patients with AR and 15 healthy controls, were evaluated for allergies to dust mites, cat and dog danders, cockroaches (Blatella germanica), molds, grasses, weeds, and tree pollen using skin prick tests. Participants were also evaluated for inflammatory conditions by measuring total blood count with differential; concentrations of rheumatoid factor, fibrinogen, and C-reactive protein; and erythrocyte sedimentation rate. CCL3 in blood samples was measured at the Immunology Laboratory, Cantacuzino National Institute for Military Medical Research and Development, Bucharest, Romania, using Human Multianalyte Profiling Base Kits (R\&D Systems Inc., Minneapolis, MN).

\section{Results}

Mean serum CCL3 concentration was significantly higher in patients with AR than in controls $(15.03 \pm 7.11$ $\mathrm{pg} / \mathrm{ml}$ vs. $8.34 \pm 4.46 \mathrm{pg} / \mathrm{ml}, \mathrm{p}=0.001$ [t-test] and $\mathrm{p}=0.026$ [Mann-Whitney test]). CCL3 concentrations correlated with polysensitization, defined as two or more positive prick tests per patient $(r=0.325, p=0.046)$ and seasonal AR $(r=0.482, \mathrm{p}=0.002)$.

Received 03/20/2020

Review began 03/26/2020 Review ended 04/17/2020 Published 04/22/2020

\section{(c) Copyright 2020}

Berghi et al. This is an open access article distributed under the terms of the Creative Commons Attribution License CC-BY 4.0., which permits unrestricted use, distribution, and reproduction in any medium, provided the original author and source are credited.

\section{Conclusions}

Elevated levels of CCL3 were seen in our patients with AR. We have observed correlations with polysensitization and seasonal allergies. These results suggest that chemokines might play an important role in the pathogenesis of AR. In the future, chemokines might be used in endotype classification of patients with AR and as a possible target in the treatment of AR.

Categories: Otolaryngology, Allergy/Immunology

Keywords: chemokine, allergic rhinitis

\section{Introduction}

Allergic rhinitis (AR) is a symptomatic condition of the nose induced by an immunoglobulin E (IgE)mediated inflammatory reactions after exposure of the nasal mucosa to an allergen. Chemokines play an important role in both the immediate and late phases of the allergic inflammatory process, inducing the activation and migration of immune system cells, including mast cells and eosinophils, into target organs, and activating macrophages to trigger B-cells to synthesize allergen specific-IgE [1]. Chemokine ligand 3 (CCL3, a macrophage inflammatory protein $1 \alpha$ ) is produced by a variety of cells, including lymphocytes, resident and recruited monocytes and macrophages, fibroblasts, and epithelial cells. CCL3 is a potent chemokine that binds to and activates lymphocytes and monocytes through its receptors chemokine receptor (CCR) 1 and CCR5. CCL3 synthesis is important in maintaining the recruitment of inflammatory cells during episodes of inflammation, as well as activating eosinophils and T-cells and regulating Ig production $[2,3]$. CCL3 constitutes a second signal for mast cell degranulation, acting as a direct costimulatory signal via CCR1. CCL3 is, therefore, essential for inducing acute-phase AR, making this chemokine critical for mast cell activation [4]. 
Several studies in humans have investigated the role of CCL3 in respiratory allergies. A pharmacological study investigating the role of the antihistamine fexofenadine found that nasal antigen challenge increased peak levels of CCL3 in nasal secretions, from $8.1 \pm 1.8 \mathrm{ng}$ to $11.2 \pm 1.7 \mathrm{ng}(\mathrm{P}=0.05)$ [5]. Levels of CCL3 increased observed six to eight hours after challenge with the nasal allergen Timothy grass pollen and decreased after treatment with fluticasone propionate, an inhibitor of Th2 cytokine synthesis [6]. The levels of CCL3 were significantly higher in patients with seasonal allergic rhinitis than in patients with perennial allergic rhinitis and healthy controls [3]. Moreover, serum concentrations of CCL3 in subjects allergic to ragweed were reduced outside the pollen season [7]. High levels of CCL3, along with high levels of the chemokines eotaxin, RANTES (Regulated upon Activation, Normal T-cell Expressed and Presumably Secreted), and monocyte chemoattractant protein-1 were observed during late responses to grass pollen [8]. Also, high levels of CCL3 messenger RNA were induced in mouse macrophage RAW 264.7 cells by birch and oak dusts, which have been associated with the development of allergic diseases [9]. The present study compared serum concentrations of CCL3 in patients with AR and healthy controls and correlated these concentrations with various aspects of respiratory allergies.

\section{Materials And Methods}

This prospective study was approved by the local medical committee of Santa Maria Clinics and Laboratories (formerly Anima Medical Center Bucharest) and the Ethics Committee for Research of Carol Davila University of Medicine and Pharmacy Bucharest. All participants provided written informed consent before enrollment.

This study enrolled 39 participants, 24 patients with AR and 15 healthy controls, all aged $>18$ years. AR was diagnosed using the 2019 updated Allergic Rhinitis and its Impact on Asthma criteria [10]. Twenty patients were diagnosed as having seasonal allergic rhinitis (SAR) and four perennial allergic rhinitis (PAR). Seasonal allergies usually occur during the spring and fall season in response to outdoor allergens like pollens. Perennial allergies can occur year round in response to indoor allergens, like dust mites, cockroaches and pet dander. Allergic sensitization was assessed according to the European Academy of Allergy and Clinical Immunology guide for the investigation of respiratory allergies [11]. All participants were subjected to skin prick tests (Lofarma, Milan, Italy) for 22 aeroallergens considered relevant to Europe, including Romania. These included dust mites (Dermatophagoides farinae and D. pteronyssinus), cockroaches (Blatella germanica), cat and dog danders, molds (Alternaria, Aspergillus, Penicillium and Cladosporium), and pollens of grasses, cereals, weeds (Ambrosia, Artemisia, Parietaria, Plantago lanceolata, and Helianthus annuus) and trees (Betulaceae, Oleaceae, Platanus occidentalis, Cupressaceae, Fagaceae, and Salicaceae). All tests were performed by an allergist, with a positive result defined as a wheal diameter $\geqslant 3 \mathrm{~mm}$ with surrounding erythema. All tests included histamine dihydrochloride $(1 \mathrm{mg} / \mathrm{ml})$ as a positive control and allergen-free saline solution as a negative control. The severity of AR was evaluated using the Total Nasal Symptom Score (TNSS), which is composed of at least three of the following four nasal symptoms: rhinorrhea, nasal congestion, nasal itching, and sneezing, with each having three levels of severity (mild, moderate and severe) [12]. Other inflammatory conditions were excluded by serologic analysis, including total blood count with differential; concentrations of rheumatoid factor, fibrinogen, and C-reactive protein; and erythrocyte sedimentation rate. Only participants with normal values were included in the study. All participants were examined by an otorhinolaryngologist using a flexible nasal fibroscope, with nasal mucosal hypertrophy (NMH) considered a sign of chronic inflammation.

CCL3 concentrations in blood samples were measured at the Immunology Laboratory of the Cantacuzino National Institute for Military Medical Research and Development Bucharest, using Human Multianalyte Profiling Base Kit A (R\&D Systems Inc., Minneapolis, MN). The measurement of CCL3 levels in serum samples was realized in our study because in our facilities (clinics and laboratory) only for blood products were possible sampling, transport, storage and analysis of the tests. The detection limit was set to $3.2 \mathrm{pg} / \mathrm{ml}$ according to manufacturer's instructions. Plates were read on a Luminex 200 platform (Luminex Corporation, Austin, TX), and data were processed with Luminex 200 IS 2.3 Star Station software (Applied Cytometry, Plano, TX) [13]. Concomitantly we have measured the serum levels of other chemokines (MCP1/CCL2 (monocyte chemoattractant protein-1), IP-10/CXCL10 (interferon gamma-induced protein 10), ENA78/CXCL5 (epithelial-derived neutrophil-activating peptide 78)) in order to obtain a wider image of the role of different subfamilies of chemokines in the pathogenesis of AR. We did not reach a statistical significance.

All statistical analyses were performed using IBM SPSS Statistics for Windows, Version 20.0. (IBM Corp., Armonk, NY) [11]. Because the groups were unbalanced, they were compared by Mann-Whitney U test groups with the Monte Carlo simulation technique and Student's t-tests (for equal variances not assumed). The correlation of variables was assessed by Pearson's or Spearman's correlation test, as appropriate. The 95\% reliability range of all data were reported, and p-values $<0.05$ defined as statistically significant.

\section{Results}

The baseline demographic and clinical characteristics of the study subjects are detailed in Table 1 . 


\section{Cureus}

\begin{tabular}{|c|c|c|}
\hline Characteristic & AR patients & Healthy controls \\
\hline Number & 24 & 15 \\
\hline Age (median), years & 33 & 39 \\
\hline Sex: M/W & 9/15 & $5 / 10$ \\
\hline $\lg \mathrm{E}$ & 116.34 & 14.99 \\
\hline Eosinophils (absolute number) (N.V. $<500$ cells per microliter of blood) & 350 & 200 \\
\hline Eosinophils (percent) (N.V. $<7 \%$ of the circulating leukocytes) & $3.4 \%$ & $1.8 \%$ \\
\hline VEMS (ml/s) & 128 & 27 \\
\hline
\end{tabular}

\section{TABLE 1: Baseline demographic and clinical characteristics of the study population}

AR: Allergic rhinitis; M/W: Men/women; IgE: Immunoglobulin E; VEMS: Maximum expiratory ventilation per second; N.V.: Normal values.

All participants lived in the city of Bucharest. Total serum IgE levels (a general marker for atopy), the absolute number and percent eosinophils (markers for hypereosinophilia), and maximum expiratory ventilation per second (an indicator of lung airway inflammation) were evaluated. CCL3 concentrations were significantly higher in AR patients than in healthy controls $(15.03 \pm 7.11 \mathrm{pg} / \mathrm{ml}$ versus $8.34 \pm 4.46 \mathrm{pg} / \mathrm{ml} ; \mathrm{p}=$ 0.001 by t-test, $\mathrm{p}=0.026$ by Mann-Whitney U-test) (Tables 2,3 and Figure 1 ).

\begin{tabular}{|l|l|l|l|l|l|}
\hline \multicolumn{1}{|c|}{ Group } & N & Mean $(\mathbf{p g} / \mathrm{ml})$ & Standard deviation & Standard error (mean) \\
\hline \multirow{2}{*}{ CCL3 } & AR patients & 24 & 15.031 & 7.116 & 1.452 \\
& Healthy controls & 15 & 8.340 & 4.465 & 1.152 \\
\hline
\end{tabular}

TABLE 2: CCL3 concentrations in AR patients and healthy controls

AR: Allergic rhinitis; CCL3: Chemokine ligand 3.

\begin{tabular}{|c|c|c|c|c|c|c|c|c|}
\hline & & \multicolumn{7}{|c|}{ t-test for equality of means } \\
\hline & & $\mathrm{t}$ & df & $\begin{array}{l}\mathrm{p}- \\
\text { value }\end{array}$ & $\begin{array}{l}\text { Mean } \\
\text { difference }\end{array}$ & $\begin{array}{l}\text { Standard error } \\
\text { difference }\end{array}$ & \multicolumn{2}{|c|}{$\begin{array}{l}95 \% \text { Confidence Interval of the } \\
\text { difference }\end{array}$} \\
\hline CCL3 & $\begin{array}{l}\text { Equal variances not } \\
\text { assumed }\end{array}$ & 3.60 & 36.990 & 0.001 & 6.69 & 1.85 & 2.93 & 10.44 \\
\hline \multicolumn{9}{|c|}{$\begin{array}{l}\text { TABLE 3: Statistical comparisons of CCL3 co } \\
\text { determined by independent samples t-tests }\end{array}$} \\
\hline \multicolumn{9}{|c|}{ AR: Allergic rhinitis; CCL3: Chemokine ligand 3; t: Student t-test; df: Degrees of freedom. } \\
\hline
\end{tabular}




\section{Cureus}

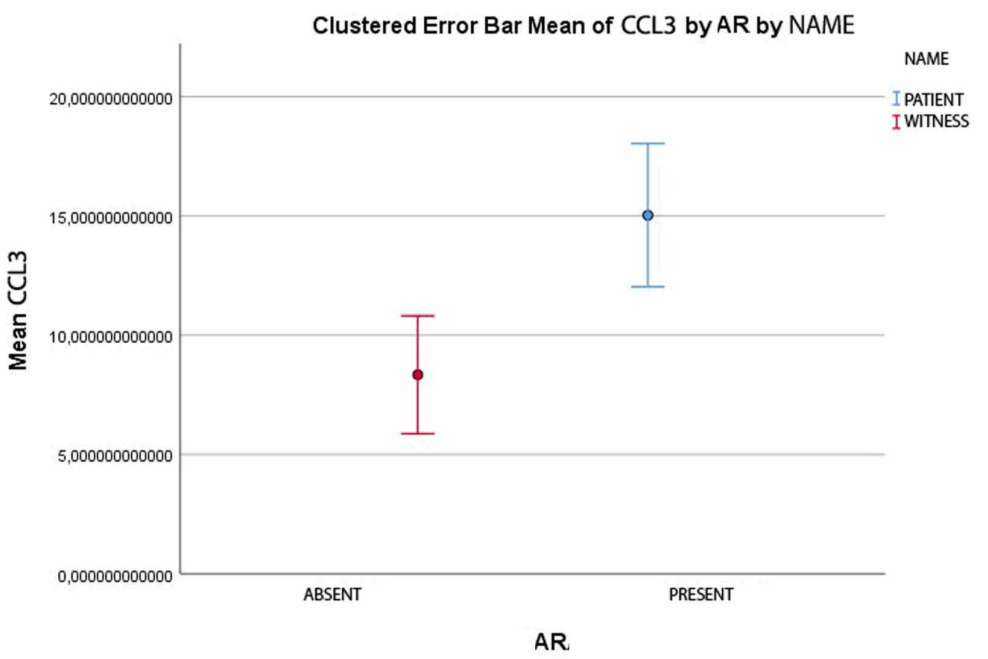

Error Bars: $95 \% \mathrm{Cl}$

FIGURE 1: CCL3 values patients versus witness

CCL3 concentrations were significantly associated with NMH ( $\mathrm{p}=0.003$ by Mann-Whitney U-test) and correlated with polysensitization (two or more positive skin prick tests per patient; $r=0.325, p=0.046$ ) and seasonal allergic rhinitis $(r=0.482, p=0.002$; Table 4$)$. The observed correlation between NMH and seasonal allergic rhinitis might be explained by the fact that although SAR has a temporal clinical picture, a subclinical inflammation is presented at cellular levels all years and becomes active when allergens take direct contact with local mast cells.

\begin{tabular}{|c|c|c|c|c|c|c|c|c|c|c|}
\hline & & Name & CCL3 & Age & AR & Duration of disease & NMH & Seasonality & Sensitization & TNSS \\
\hline \multirow{3}{*}{$\mathrm{Nam}$} & Pearson Correlation & 1 & $-.472^{* *}$ & .177 & $-1.000^{* *}$ & $-.532^{* *}$ & $-.5566^{* *}$ & $-.936^{* *}$ & $-.948^{* *}$ & $-.741^{*}$ \\
\hline & Sig. (2-tailed) & & .002 & .072 & .000 & .000 & .000 & .000 & .000 & .000 \\
\hline & & & & & & & & & & \\
\hline \multirow[b]{3}{*}{ CCL3 } & Pearson Correlation & $-.472^{* *}$ & 1 & $.340^{*}$ & $.472^{* *}$ & $.346^{*}$ & $.570^{* *}$ & $.482^{* *}$ & $.330^{*}$ & $.495^{* *}$ \\
\hline & Sig. (2-tailed) & .002 & & .034 & .002 & .031 & .000 & .002 & .040 & .001 \\
\hline & & & & & & & & & & \\
\hline \multirow[b]{3}{*}{ Age } & Pearson Correlation & .177 & $.340^{*}$ & 1 & -.177 & -.102 & -.027 & -.154 & $-.216^{*}$ & $-.298^{* *}$ \\
\hline & Sig. (2-tailed) & .072 & .034 & & .072 & .301 & .782 & .117 & .027 & .002 \\
\hline & & & & & & & & & & \\
\hline & Pearson Correlation & $-1.000^{* *}$ & $.472^{* *}$ & -.177 & 1 & $.532^{* *}$ & $.556^{* *}$ & $.936^{* *}$ & $.948^{* *}$ & $.741^{* *}$ \\
\hline & Sig. (2-tailed) & .000 & .002 & .072 & & .000 & .000 & .000 & .000 & .000 \\
\hline
\end{tabular}

AR 


\section{Cureus}

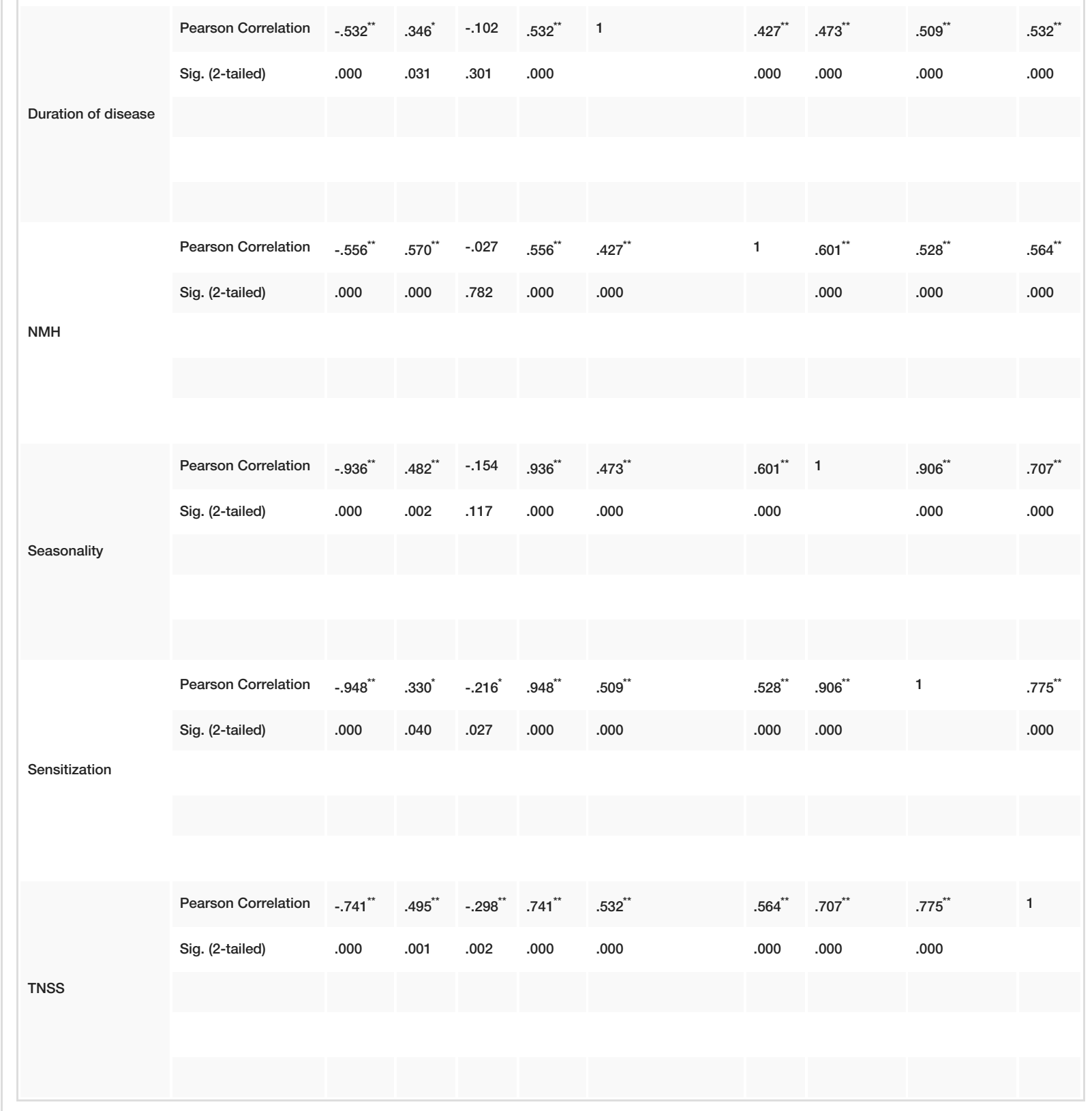

\section{TABLE 4: Correlations of parameters in this study}

AR: Allergic rhinitis; NMH: Nasal mucosal hypertrophy; TNSS: Total nasal symptom score; CCL3: Chemokine ligand 3.

CCL3 was also associated with sensitization to grass pollen $(\mathrm{p}=0.040)$ and with patient age $(\mathrm{r}=0.495, \mathrm{p}=$ 0.012).

\section{Discussion}

Our finding that serum CCL3 concentrations are higher in patients with AR than in healthy controls is in good agreement with CCL3 concentrations in nasal secretions [3]. The latter results are likely closer to real situations, in that chemokine concentrations were measured directly at the site of allergic reaction rather than in blood samples. Serum CCL3 concentrations may have been affected by systemic inflammatory conditions. To minimize this possibility, we measured important markers of systemic inflammation, including total blood count with differential; concentrations of rheumatoid factor, fibrinogen, and Creactive protein; and erythrocyte sedimentation rate, and excluded all patients and controls with abnormal results. Measurement of CCL3 and other chemokine levels in nasal secretions is, by far, the most sensitive and specific method to evaluate their presence in allergic rhinitis. But this demands a specialized stuff (nurse, physicians (otorhinolaryngologist), laboratory personal) that is limited to university/research facilities. Measurement of chemokines in serum samples may be performed in many ordinary laboratories 
around the globe. This technique is affected by the fact that levels of chemokines might be influenced by other systemic conditions.

The association between CCL3 concentration and NMH confirms that CCL3 plays a role in the pathogenesis of chronic inflammation, including respiratory allergies [2, 3]. Moreover, our finding of an association between CCL3 and grass pollen allergy is in agreement with previous results [6, 9]. CCL3 concentration appears to be associated with pollen sensitization, but this observation requires confirmation in future studies [3, 6, 7]. To our knowledge, however, no previous study had reported a correlation between CCL3 concentration and polysensitization to aeroallergens, providing further evidence of the role of CCL3 in the pathogenesis of AR. Several studies have suggested a role for CCL3 in allergen sensitization [3, 6, 7].

The CCL3 gene may be a selective end-point marker in CD34+-progenitor derived dendritic cells exposed to contact allergens and irritants, enabling these cells to differentiate between chemical sensitizers and irritants [14]. We also observed a correlation between CCL3 concentration and TNSS $(r=0.495, \mathrm{p}=0.001)$. The mean TNSS score in the group of 24 patients was $7.0 \pm 3.83$, indicating moderate disease. This may indirectly suggest that CCL3 plays a role in the pathogenesis of AR.

Our study has two major limitations. First, the study population was relatively small, which might have affected the statistical power of the study. Secondly, while we tried to eliminate all systemic inflammatory conditions that might influence the levels of CCL3 by using the most important inflammatory markers and keeping only participants with healthy values, we could not exclude a possible infection or inflammation in one or more patients, which constitutes the second major limitation of our study.

\section{Conclusions}

Our study intended to evaluate the role of CCL3 in the pathogenesis of AR. CCL3 presented significant differences between patients with AR and healthy controls. We have shown that the measurement of the levels of CCL3 in blood samples might represent an alternative approach of evaluating chemokines in allergic rhinitis in a non-academic outpatient facility when nasal collection of probes is not feasible. CCL3 also correlated with some aspects of respiratory allergies, such as polysensitization, seasonal allergies, and nasal mucosal hypertrophy. These findings indicate that CCL3 may play a role in AR. Our study implicated a small number of patients and evaluated CCL3 only from blood samples, and therefore, our results should be interpreted with caution. Future studies including a larger number of participants and samples from both the nose and blood may be necessary to confirm the association between CCL3 and AR.

\section{Additional Information}

\section{Disclosures}

Human subjects: Consent was obtained by all participants in this study. Avizul Comisiei de Etica a Cercetarii Stiintifice UMF Carol Davila Bucharest Cod: PO-35-F-03 issued approval 103/05.12.2016. Animal subjects: All authors have confirmed that this study did not involve animal subjects or tissue. Conflicts of interest: In compliance with the ICMJE uniform disclosure form, all authors declare the following: Payment/services info: All authors have declared that no financial support was received from any organization for the submitted work. Financial relationships: All authors have declared that they have no financial relationships at present or within the previous three years with any organizations that might have an interest in the submitted work. Other relationships: All authors have declared that there are no other relationships or activities that could appear to have influenced the submitted work.

\section{References}

1. Stylianou E, Ueland T, Borchsenius F, et al.: Specific allergen immunotherapy: effect on IgE, IgG4 and chemokines in patients with allergic rhinitis. Scand J Clin Lab Invest. 2016, 76:118-127. 10.3109/00365513.2015.1110856

2. Schaller TH, Batich KA, Suryadevara CM, Desai R, Sampson JH: Chemokines as adjuvants for immunotherapy: implications for immune activation with CCL3. Expert Rev Clin Immunol. 2017, 13:10491060. 10.1080/1744666X.2017.1384313

3. König K, Klemens C, Eder K, Nicoló MS, Becker S, Kramer MF, Gröger M: Cytokine profiles in nasal fluid of patients with seasonal or persistent allergic rhinitis. Allergy Asthma Clin Immunol. 2015, 11:26. 10.1186/s13223-015-0093-x

4. Miyazaki D, Nakamura T, Toda M, Cheung-Chau KW, Richardson RM, Ono SJ: Macrophage inflammatory protein-1alpha as a costimulatory signal for mast cell-mediated immediate hypersensitivity reactions. J Clin Invest. 2005, 115:434-442. 10.1172/JCI18452

5. Bensch GW, Nelson HS, Borish LC: Evaluation of cytokines in nasal secretions after nasal antigen challenge: lack of influence of antihistamines. Ann Allergy Asthma Immunol. 2002, 88:457-462. 10.1016/S10811206(10)62382-4

6. Erin EM, Zacharasiewicz AS, Nicholson GC, et al.: Topical corticosteroid inhibits interleukin-4, -5 and -13 in nasal secretions following allergen challenge. Clin Exp Allergy. 2005, 35:1608-1614. 10.1111/j.13652222.2005.02381.x

7. Kostova Z, Batsalova T, Moten D, Teneva I, Dzhambazov B: Ragweed-allergic subjects have decreased serum levels of chemokines CCL2, CCL3, CCL4 and CCL5 out of the pollen season. Cent Eur J Immunol. 2015, 


\section{Cureus}

40:442-446. 10.5114/ceji.2015.56965

8. Cockcroft DW, Scadding GW, Gunawardana N: Bronchial and nasal challenge testing. Middleton's Allergy: Principles and Practice. Elsevier, 2020. 1:1015-1038.

9. Määttäa J, Majuria M-L, Luukkonen R, Lauerma A, Husgafvel-Pursiainen K, Alenius H, Savolainen K: Characterization of oak and birch dust-induced expression of cytokines and chemokines in mouse macrophage RAW 264.7 cells. Toxicology. 2005, 215:25-36. 10.1016/j.tox.2005.06.021

10. Klimek L, Bachert C, Pfaar O, et al.: ARIA guideline 2019: treatment of allergic rhinitis in the German health system. Allergo J Int. 2019, 28:255-276. 10.1007/s40629-019-00110-9

11. Bousquet J, Heinzerling L, Bachert C, et al.: Practical guide to skin prick tests in allergy to aeroallergens . Allergy. 2012, 67:18-24. 10.1111/j.1398-9995.2011.02728.x

12. Mahatme MS, Dakhale GN, Tadke K, Hiware SK, Dudhgaonkar SD, Wankhede S: Comparison of efficacy, safety, and cost-effectiveness of montelukast-levocetirizine and montelukast-fexofenadine in patients of allergic rhinitis: a randomized, double-blind clinical trial. Indian J Pharmacol. 2016, 48:649-653. 10.4103/0253-7613.194854

13. Tang H, Panemangalore R, Yarde M, Zhang L, Cvijic ME: 384-Well multiplexed luminex cytokine assays for lead optimization. J Biomol Screen. 2016, 21:548-555. 10.1177/1087057116644164

14. Verheyen GR, Schoeters E, Nuijten J-M, et al.: Cytokine transcript profiling in CD34+-progenitor derived dendritic cells exposed to contact allergens and irritants. Toxicol Lett. 2005, 155:187-194.

10.1016/j.toxlet.2004.09.014 